\title{
Universal Aspects of Dynamic Fracture in Brittle Materials
}

\author{
Ariel Livne and Jay Fineberg \\ The Racah Institute of Physics, The Hebrew University of Jerusalem, Givat Ram, Jerusalem \\ 91904, Israel
}

\begin{abstract}
We present an experimental study of the dynamics of rapid fracture in brittle amorphous materials. In this study we utilize a class of model materials, polyacrylamide gels, in which the relevant sound speeds can be reduced by 2-3 orders of magnitude compared to "standard" brittle materials. We first demonstrate that dynamic fracture in polyacrylamide gels has characteristic features which are identical to those observed in well-studied brittle materials, such as brittle plastics and glasses. These features include the existence of a critical velocity beyond which frustrated crack branching occurs (Fineberg [1], Sharon [2]) and the profile of the micro-branches formed. We then examine the behavior of the crack fronts, which are the 1D fronts defined by the leading edge of a propagating crack. During fracture, a crack front can be locally perturbed by either an externally introduced inclusion or, dynamically, by the generation of a micro-branch. Comparison of the behavior of the excited fronts in both gels and in soda-lime glass reveals that, once again, many aspects of the dynamics of these excited fronts are the same in both materials. These include both the generation of coherent, localized waves ([Morrissey [3], Ramanathan [4], Sagy [5], Sharon [6]) ("front waves") which propagate along the crack front as well as the appearance of crack front inertia. Crack front inertia is embodied by a "memory" effect of the crack front in which directed roughly periodic lines of spatially localized micro-branches are generated. These lines are aligned in the direction of propagation (Fineberg [7], Sharon [8]) and the spacing between successive microbranches is proportional to their width (in the direction normal to the propagation direction). This scaling is identical in both glass and gels.
\end{abstract}

\section{INTRODUCTION}

We present a detailed experimental study of rapidly moving cracks in polyacrylamide gels. The dynamic behavior of cracks in these "model" materials is then compared to the corresponding dynamics of rapid fracture in both the brittle polymer, PMMA, as well as soda-lime glass. Our motivation in these experiments is two-fold. Many of the effects that we shall examine here have been quantitatively observed in either a single material or, at most, two different materials. In order to determine the degree of universality of these effects, there is, therefore, a need to perform analogous experiments in qualitatively different materials. These new experiments will allow us to examine both the premise of 
universal qualitative phenomena in dynamic fracture as well as to what degree these phenomena obey quantitative scaling relations. This is our primary motivation. Our secondary motivation is to develop an experimental system in which we can overcome experimental limitations that impede our ability to go beyond the technical barriers that are imposed by the relatively high propagation velocities that are characteristics of "standard" laboratory materials. In standard materials the propagation of rapid cracks is on the order of the Rayleigh wave speed, $V_{R}$, which is generally quite high (e.g. $3500 \mathrm{~m} / \mathrm{s}$ in glasses). In addition, any induced changes in the stress fields surrounding a crack's tip occur within shear wave propagation times. Thus, in "standard" materials the quantitative study of dynamic fracture processes is, in general, limited by the fact that all diagnostics and measurements must be extremely rapid. Since the fracture properties of a given material are largely determined by the form of the stress field surrounding the crack's tip (e.g. by the nature of the process zone), we are faced with the problem of visualizing small regions surrounding the crack tip at high spatial resolution and at $\mu \mathrm{sec}$ time scales.

These technical problems can be largely circumvented by conducting experiments on polyacrylamide gels. In these materials it is possible to reduce the elastic constants by 4-5 orders of magnitude, relative to standard brittle materials. This leads to a corresponding reduction of 2-3 orders of magnitude of the Rayleigh wave speeds, hence slowing down the fracture process to speeds which are amenable for study by means of fast video cameras, enabling both high spatial and temporal resolution. These materials can be tuned to be both brittle and amorphous. They are sufficiently different from more standard materials in both their molecular structure and mechanical properties so as to enable us to perform both qualitative and quantitative evaluations of the universal features of rapid fracture. Polyacrylamide gels have the following material properties:

1. The shear wave speeds, hence Rayleigh wave speeds, in these materials can be tuned over a wide range. In our experiments $V_{R}$ was varied between $1-20 \mathrm{~m} / \mathrm{s}$.

2. The macroscopic properties of these gels can be tuned to model either materials with either highly brittle or more plastic, ductile behavior, by simple changes to their chemical composition. Both the elastic and plastic properties of these gels can be tuned by varying both the polymer and cross-linker concentrations used to form them.

\section{COMPARISON OF FRACTURE IN GELS TO OTHER MATERIALS}

We performed a detailed study of both the dynamic behavior and resulting characteristic features on and beneath the fracture surface. We then compared these measurements to corresponding experiments in brittle glass that were performed in our laboratory. As we shall demonstrate, these comparisons indicate 
that precisely the same dynamics occur in both materials. This establishes this system as one that is able to provide both qualitative and quantitative information regarding rapid fracture processes.

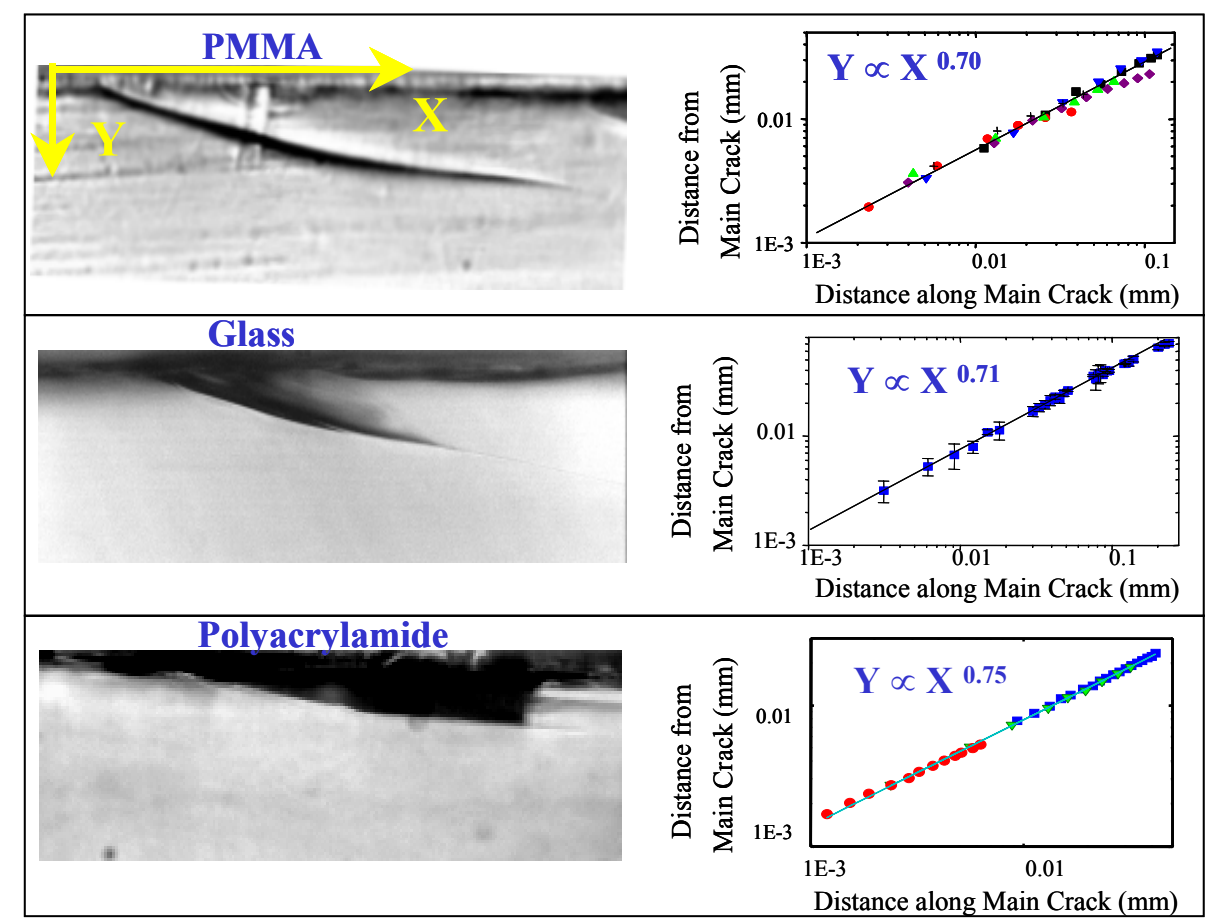

Figure 1: A comparison of micro-branches in glass, PMMA and gels. Photographs of typical micro-branches in these three materials (left) and (right) the functional forms of micro-branch profiles - from a number of samples in each material. In each material, the superimposed measurements taken from different samples all trace out the same curve. This functional form is identical in all of the three materials, behaving as $\mathrm{y} \propto \mathrm{x}^{0.7}$, where $\mathrm{x}$ is the direction of propagation and $\mathrm{y}$ the direction normal to the fracture surface.

We will present data that substantiates the equivalence of the fracture processes in both "real" brittle materials and brittle polyacrylamide gels. The following data will show:

1. Micro-branching (microscopic secondary crack branches) occurs in PMMA, glass and gels at a critical velocity of about $40 \%$ of the Rayleigh wave speed. Below this velocity the fracture surface is featureless and mirror-like and only a single crack will propagate. 
2. Direct observation of the crack tip in gels shows that micro-branches occur as "tip-splitting" instabilities of the crack tip and are not observed to be the result of void coalescence.

3. The overall profiles of the micro-branches are identical to those observed in brittle polymers and glass (See e.g. Figure 1).

\section{THREE DIMENSIONAL EFFECTS IN FRACTURE}

The tip of a rapidly propagating crack is generally idealized as a point-like singular object which runs through a $2 \mathrm{D}$ material. In a "real" $3 \mathrm{D}$ material, this view can be formally used to describe a crack's motion as long as the onedimensional front, defined by leading edge of the crack, is translationally invariant in the direction normal to propagation. Once this invariance is broken, however, this one-dimensional "crack front" can be excited (Morrissey [3], Ramanathan [4]). Experimentally, we find that once the translational invariance along a "straight" crack front is broken, the front can indeed lose its stability. This crack front instability generates fractographic features which are characteristic of dynamic fracture in both highly brittle "ideal" materials, such as glass (Sharon [6]) and polyacrylamide gels, as well as "non-ideal" highly inhomogeneous materials such as brittle rock (Sagy [5]). We will demonstrate that:

- Micro-branches are formed along directed lines ("branch-lines") that are both oriented in the direction of the fracture propagation and spatially confined in the direction normal to the propagation direction (Sharon [7], Fineberg [8]).

- The existence of crack front waves. Localized elastic waves are generated by the interaction of the crack front with a spatially localized disturbance. These disturbances can be either a material inhomogeneity (e.g. an inclusion within the material) or a micro-branching event. These waves ("front waves") propagate along the crack front.

- An intrinsic periodicity of micro-branches within a "branch-line" exists (Sharon [7], Fineberg [8]). This periodicity scales in the same way in both glass and gels (Figure 2). Both the existence of "branch-lines" and their internal periodicity indicate that, upon the loss of translational invariance along the crack front, a crack develops inertia. In other words, although a crack in an effectively two-dimensional medium behaves as a "massless" entity, once this effective two-dimensionality is broken, a crack is aware of its past history. One important result of this is that the energy flow along a crack front becomes inhomogeneously distributed once the translational invariance along the front is broken. This would then tend to localize the damage distribution in the $\mathrm{z}$ direction (see Figure 2) along a rapidly moving crack front. 


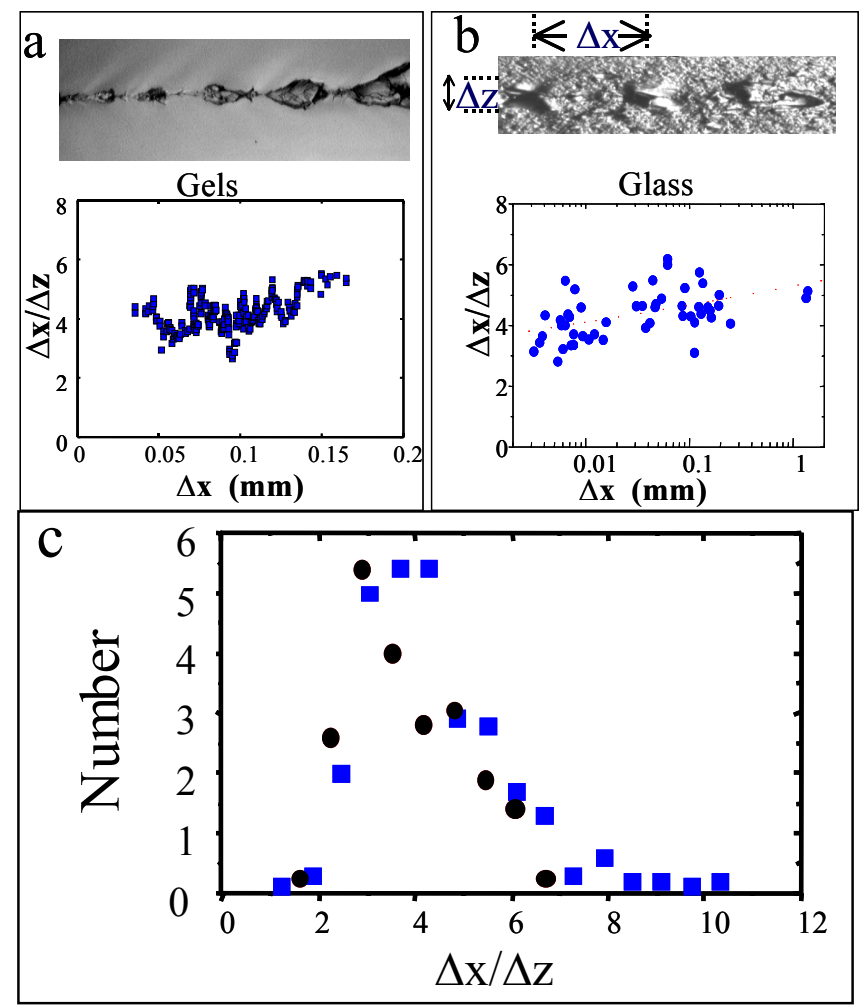

Figure 2: The inertia of cracks and the periodicity of micro-branches. In both gels (a) and glass (b) The spacing, $\Delta x$, between successive micro-branches in the direction of propagation scales with their width, $\Delta z$. The scaling is identical in both materials as the ratio $\Delta \mathrm{x} / \Delta \mathrm{z}$ indicates in (a) and (b). Surprisingly, even the distribution of this ratio (c) is nearly identical in gels (circles) and glass (squares).

In summary, we show that many features of the dynamic fracture of polyacrylamide gels are both qualitatively and quantitatively identical with those in "real" materials. This universality of behavior indicates that these features are material independent and, therefore, characteristics of the fracture process in brittle, amorphous materials. Having established polyacrylamide gels as materials whose dynamic behavior is representative of brittle fracture, we are now in a position to utilize the unique properties of these gels (i.e. the possibility of slowing down characteristic dynamic processes by nearly three orders of magnitude) to study processes (e.g. the structure and dynamic behavior of the process zone) that have, so far, been technically impossible to visualize. 


\section{REFERENCES}

1. Fineberg, J., and M. Marder, Instability in dynamic fracture, Physics Reports, 313 (1-2), 2-108, 1999.

2. Sharon, E., and J. Fineberg, Microbranching Instability and the Dynamic Fracture of Brittle Materials, Physical Review B54 (10), 7128-7139, 1996.

3. Morrissey, J.W., and J.R. Rice, Crack front waves, Journal of the Mechanics and Physics of Solids, 46 (3), 467-87, 1998.

4. Ramanathan, S., and D.S. Fisher, Dynamics and instabilities of planar tensile cracks in heterogeneous media, Physical Review Letters, 79 (5), 877-880, 1997.

5. Sagy, A., Z. Reches, and J. Fineberg, Dynamic fracture by large extraterrestrial impacts as the origin of shatter cones, Nature, 418 (6895), 310-313, 2002.

6. Sharon, E., G. Cohen, and J. Fineberg, Propagating solitary waves along a rapidly moving crack front, Nature, 410 (6824), 68-71, 2001.

7. Sharon, E., G. Cohen, and J. Fineberg, Crack front waves and the dynamics of a rapidly moving crack, Physical Review Letters, 88 (8), 2002.

8. Fineberg, J., E. Sharon, and G. Cohen, Crack front waves in dynamic fracture, International Journal of Fracture, 119 (3), 247-261, 2003. 